\title{
A New Endoscopic Appearance of Collagenous Colitis
}

Collagenous colitis is a chronic diarrheal illness of indeterminate etiology that typically presents in the late sixth or early seventh decade of life (1). The physical examination and laboratory studies are normal or nonspecific, with fecal leukocytosis being the only abnormality found in the majority of patients tested (2). This report describes a patient with collagenous colitis and previously unrecognized colonoscopic features.

A 65-year-old man was referred to our department due to watery diarrhea, which had persisted for the previous six months. Bowel movements occurred six to eight times a day, and were not associated with abdominal pain. The physical examination, biochemical and hematological investigations, stool cultures, and stomach, small intestine, and colonic barium examinations were normal. Duodenal biopsy samples were unremarkable.

Colonoscopic examination revealed a normal appearance of the mucosa up to the transverse colon. On the right part, mainly in the lower part of the ascending colon, multiple red spots were seen (Figure 1). In these areas, the normal vascular pattern had diminished. Several biopsy specimens were obtained from both pathological and normal areas. Histological examination of the biopsies from the pathological area showed a diffuse and marked collagen band below the surface epithelium (Figure 2). The patient's diarrhea responded after two weeks to treatment with oral methylprednisolone $(16 \mathrm{mg} /$ day $)$, and he has now been free of symptoms for three months.

Patients with collagenous colitis have either an endoscopically normal colon or minor nonspecific abnormalities, such as edema and mild opalescence of the colonic mucosa (3). A case of collagenous colitis was recently reported in which the normal haustrations were obliterated, and the right colon showed multiple hemorrhagic lacerations (4). In another case, a woman with diarrhea was found to have a $5-\mathrm{cm}$ square of finely nodular mucosa in the right colon, suggestive of a flat villous adenoma. However, it was argued that the biopsies did not reveal adenoma but collagenous colitis (5). Endoscopic examination is limited to the rectosigmoid colon in most collagenous colitis series, and proximal lesions may therefore go undetected. The findings in the present case and in others recently reported emphasize the importance of total colonoscopy and colonic biopsies rather than isolated rectosigmoid biopsies in patients with unexplained diarrhea.

P. Katsinelos ', I. Katsos ', K. Patsiaoura ', P. Xiarchos' ', I. Goulis ', N. Eugenidis ${ }^{2}$

${ }^{1}$ Dept. of Gastroenterology, Theagenion Hospital, Thessaloniki, Greece;

${ }^{2}$ Second Propedutic Clinic, Aristotelion University, Thessaloniki, Greece

\section{References}

1. Zins BJ, Sandbory WJ, Tremaine WJ. Collagenous and lymphocytic colitis: subject review and therapeutic alternatives. Am J Gastroenterol 1995; 90: 1394-1400.

2. Lawson JM, Wolosin J, Mottet MD, et al. Collagenous colitis: an association with fecal leukocytes. J Clin Gastroenterol 1988; 10: $672-5$.

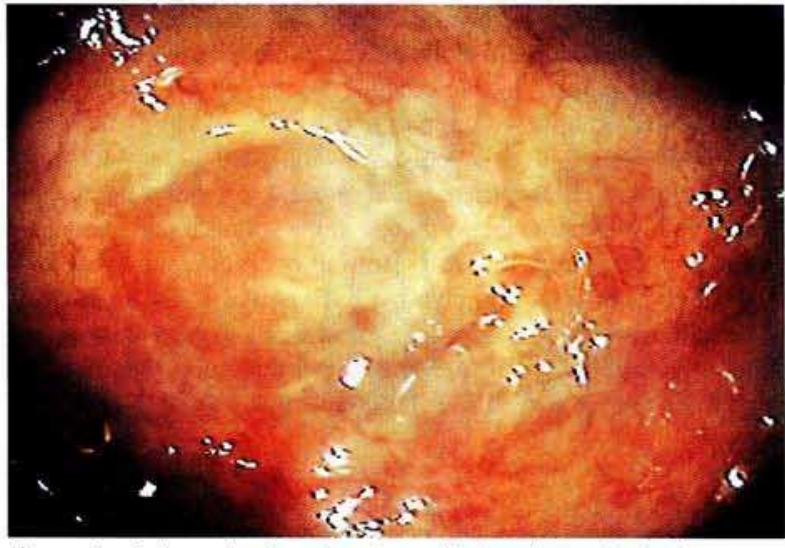

Figure 1: Endoscopic view, showing multiple red spots in the lower part of the ascending colon.

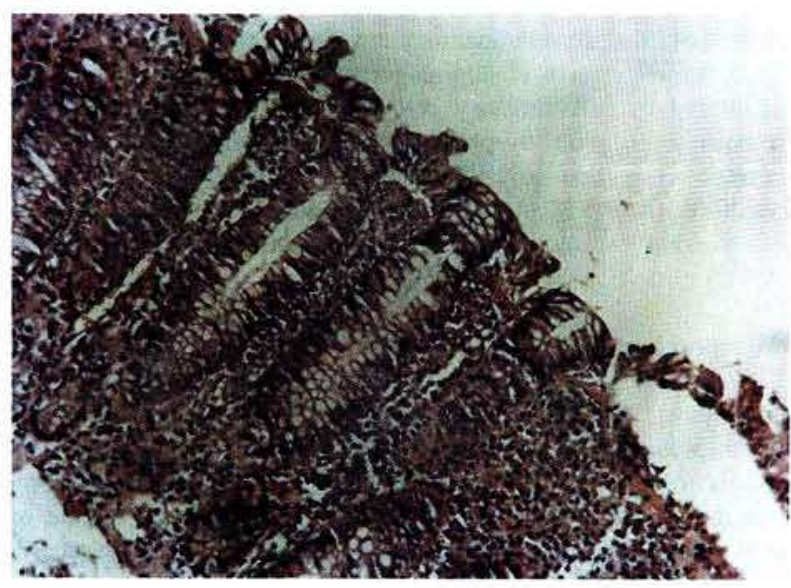

Figure 2: Thickened subepithelial collagen layer, consistent with collagenous colitis, and a moderate infiltrate in the lamina propria (hematoxylineosin, $\times 250$ ).

3. Silverstein FE, Tytgat GNJ. Collagenous colitis. In: Atlas of Gastrointestinal Endoscopy. New York: Gower Medical, 1987.

4. Richieri JP, Bonneau HP, Cano N, Costanzo JD, Nartin J. Collagenous colitis: an unusual endoscopic appearance. Gastrointest Endosc 1993; 39: 192-96.

5. Smiley DN, Barkin J. Unusual endoscopic appearance of collagenous colitis. J Clin Gastroenterol 1993; 17: 84-5.

Corresponding Author

P. Katsinelos, M.D.

Ydras 31

TT 54638 Thessaloniki

Greece 